\title{
P79 Non-invasive Estimation of Central Pressures in Abdominal Aortic Aneurysm Patients and Changes in PWV After EVAR
}

\author{
Suzanne Holewijn ${ }^{1, *}$, Lennart van de Velde ${ }^{2,3}$, Jenske Vermeulen ${ }^{4,3}$, Michel Reijnen ${ }^{3,2}$ \\ ${ }^{1}$ Rijnstate, Vascular Research Center, Arnhem, Netherlands \\ ${ }^{2}$ Multimodality Medical Imaging Group, Technical Medical Centre, University of Twente, Enschede \\ ${ }^{3}$ Rijnstate, Department of Vascular Surgery, Arnhem, Netherlands \\ ${ }^{4}$ RadboudUMC, Nijmegen, Netherlands
}

\section{ABSTRACT}

Background: Abdominal aortic aneurysm (AAA) disease is mainly treated by endovascular aneurysm repair (EVAR) and PWV has been shown to increase after EVAR. Arterial stiffness is predictive of cardiovascular disease, but has not been investigated in AAA-patients. We investigated if central pressures (CP) can be estimated correctly in AAA-patients and changes over time in pulse wave velocity (PWV), since PWV-changes during follow-up are currently unknown.

Methods: Non-invasive (SphygmoCor) and invasive waveform recordings were performed simultaneously before and after EVAR. A generalized ascending-to-abdominal aorta transfer function (GTFAA) was used to estimate CP from the invasively measured abdominal aorta pressure-waveform, and compared to the non-invasively estimated CP. Changes in PWV were evaluated compared to baseline (6-weeks and 1-year).

Results: The mean difference between invasive and non-invasive central systolic pressure was $-11.5 \mathrm{mmHg}$ (limits-ofagreement: $-63.5 ; 40.6)$ before and $0.03(-33.5 ; 33.6)$ after implant. For central diastolic and mean pressure mean difference was $-12.2(-39.3 ; 14.8)$ and $-9.6(-45.3 ; 26.1)$, respectively. Median (IQR) PWV was $10.5(9.2 ; 13.2)$ at baseline, $10.5(10.1 ; 12.4)$ at discharge, $11.5(10.6 ; 12.9)$ at 6 weeks, and $11.3(9.9 ; 13.0)$ at 1 year follow-up without significant differences over time. Increases in $\mathrm{CP}$ were equal to peripheral pressure increases.

Conclusion: Synthesizing CP with non-invasive measurements in combination with the use of a GTFAA in patients with AAA is feasible especially after EVAR. No changes over time in PWV were observed. Current ongoing analyses include evaluation by prosthesis type, wave type, and influence of intra-luminal thrombus (NCT01220245).

(c) 2019 Association for Research into Arterial Structure and Physiology. Publishing services by Atlantis Press International B.V. This is an open access article distributed under the CC BY-NC 4.0 license (http://creativecommons.org/licenses/by-nc/4.0/). 